\title{
"Trade-Off between Detection and Resolution of Two Point Objects Under Various Conditions of Imaging Situations: Part-I: Mathematical Formulation of the Problem"
}

\author{
P. Thirupathi \\ Department of Mathematics, Osmania University, Hyderabad, 500007, A.P, India.
}

\begin{abstract}
It is a well-experienced fact that whenever one tries to detect a weak object point in the vicinity of an intense point object, viz., a binary star-SIRUS and its companion weak satellite star, there is always loss of resolution of the optical system. In other words, one wants to improve the defectively of the system, there is always a loss of resolution capabilities of the system. Thus, there is a trade-off between Detection and Resolution of optical systems under various imaging situations. In this first paper of discussion of this trade-off, we have derived the Fourier analytical formulation of this problem. This formulation will be used to find out a compatible trade-off between Detection and Resolution in our further publications.
\end{abstract}

Key words: Fourier Optics, Mathematical Optics, Super-Resolution, etc.-

\section{Introduction}

In this paper, the two-point resolution capabilities have been discussed for an optical system with parabolic filters. The Rayleigh and the Sparrow resolution limits are computed and studied as functions of the degree of coherence of illumination, (Incoherent, coherent and partially coherent) of the two point objects. The problem of the definition and determination of an image quality criterion has long been and still is a major one in the field of image evaluation and assessment. Though a number of physical parameters for assessing the quality of an image have been proposed from time to time, none of these measures is completely satisfactory. Some of these parameters are: - Resolving power, Strehl Definition, Optical Transfer Function, Equivalent Passband, Relative Structural Content, Correlation Quality. Image Fidelity and peachiness. Historically, one of the first measures established for the evaluation of optical system was to specify how well the system could resolve a two-point object, and the two-point resolution is one of the simplest quality criteria in terms of the impulse response from among several criteria available is chosen. The intensity distribution in the image should satisfy the requirement of the criteria chosen. The limiting separation thus determined gives the imaging system's response in terms of two-point resolution. In the case of sources of short wave length radiations such as X-rays, gamma rays or sub-atomic particles, conventional method of ray bending i.e., reflection, refraction and diffraction, cannot be used for imaging due to their high penetrating power and rectilinear propagation. In such cases, coded imaging (CI) techniques can play an important role in determining source location and source distribution. According to them "CI", when reduced to the basics, is a two step process.

In the first step, the source information is recorded or encoded by geometrical shadow casting through a coded aperture (no ray bending is involved). In the second step, the image is matched to the coded aperture design. Though the two-point resolution is one of the simplest criterion to assess the performance of optical imaging systems, it has its inherent complexity owing to the fact that the limit of resolution is sensitive to a large number of factors via., nature of the optical system, nature of illumination, object point separation, intensity ratio of the object points degree of coherence, resolution criterion used, etc. Therefore, there has to be some flexibility in the exact quantitative definition of the limiting resolving power achievable. The importance of twopoint resolution lies in the fact that it is one of the earliest and simplest physical parameters used to evaluate the performance of optical imaging system in various imaging situations, incoherent, partially coherent and coherent illuminations. It may be noted that the resolving power of an imaging system as determined by the Rayleigh criterion is not the property of the system alone but also of the pair of objects and the coherence condition of illumination. Though the optical transfer function as an assessment parameter is superior to twopoint resolution for optical systems operating in incoherent illumination, it should be noted that partially coherent and coherent imaging system become non-linear in both amplitude and intensity, if the detection step is also included in the imaging system. Due to the non-linearity associated with the partially coherent imaging systems, the systems become object-dependent and cannot be completely characterized by a system transfer function as in the linear case. 


\section{Various Resolution Criteria}

As the subject of two-point resolution is sensitive to a wide variety of factors, a criterion of resolution is required in order to determine the limit of resolution. Several criteria have been proposed from time to time. It should be mentioned that all criteria of resolution are arbitrary and as BARAKAT [1] has mentioned, any criterion of resolution is not a law of physics. It may be pointed out that none of these criteria either determines or sets an absolute limit on the limit of resolution. Therefore, it is meaningless to talk about the absolute resolving- power of an imaging system. The resolving-power, as RONCHI [2] opined, depends on a three-fold combination of

- The source and its energy,

- The instrument and its energy distribution capacities and

- The receiver and its sensitivity characteristics.

However, arbitrary these criteria may be, they serve the purpose of comparison of the performances of various imaging systems. The criteria "yield useful rules of thumb for engineering practice". In this section , the various resolution criteria proposed from time to time are presented and the Rayleigh and Sparrow criteria of resolution that have been used in the present study are explained in more details in the following two sections. The subject of two-point resolution starts with the celebrated Rayleigh criterion. LORD RAYLEIGH [3] developed the first resolution criterion, which now bears his name. Rayleigh recognized the arbitrariness of the criterion. In his own words, "This rule is convenient on account of its simplicity and it is sufficiently accurate in view of the necessary uncertainty as to what is meant by resolution". Rayleigh criterion, through arbitrary, has the virtue of being particularly uncomplicated.

SPARROW gave the alternate criterion which he called "undulation condition". ASAKURA [4] recognizing that the case of object points having equal intensity is rare in actual imaging situations, introduced the "modified Sparrow criterion" to suit actual imaging situations and studied the problem of two-point resolution of unequally bright points under partially coherent illumination. BHATNAGAR, SIROHI and SHARMA [5] proposed a criterion for the case of unequally bright points. This criterion is based on the ratio of $I_{L P}$ (lower peak intensity) and $I_{D i p}$ (dip point intensity) in the resultant intensity distribution of the image of two points. According to this criterion, the two points are just resolved if

$$
\frac{I_{D i p}}{I_{L P}}=0.735 \text { or } \frac{L_{L P}-I_{D i p}}{I_{L P}}=0.265
$$

response functions of the optical system. In the field of image quality assessment, the study of two-point resolution is still a subject of great interest. In the field of astronomy, the problem of two-point resolution is very significant when it comes to resolve two closely spaced point objects, viz., binary stars. According to the principles of geometrical optics, there is no upper limit to the resolving power of a perfect optical imaging system. But, whatever, and perfect the optical imaging system may be, the image of a point is never a point, due to diffraction and aberration. Due to the wave nature of light, the image of a point object is a spread of light called the Fraunhofer diffraction pattern. When two object points are close to each other, the image of two-point objects consists of super-position of the two point spread functions corresponding to the two objects points and the type of super- position depends on the phase correlation between the two objects points. If the object pointes are very close to each other, it will be difficult to recognize the two images in the super-position of the point spread functions. The overlapping of individual diffraction patterns makes it a difficult job to detect the presence of individual images. The size of the finest detail or minimum separation object points that can be just resolved is given by the "Limiting Resolution". The reciprocal of the limit of resolution gives the resolving power of the optical system. The lesser the limit of resolution, the greater will be the resolving power of the optical system. In order to study the problem of two-point resolution experimentally, two pinholes in an opaque screen are illuminated by a source of monochromatic light. The light emerging from the pinholes is passed through the optical system under study. The total intensity distribution in the image of the pinholes is studied as a function of the image plane co ordinates. To determine the minimum resolvable separation between the pinholes, a criterion of distance in object space to the first null in the axial intensity distribution.

However, the Rayleigh and Sparrow criteria have been the most extensively used criteria in the field of image science. It may be mentioned that these criteria are based, directly or indirectly, on the (PSF)

The reasons for choosing these two criteria for the present dissertation are given below.

- In the field of image science, both the Rayleigh and Sparrow criteria have been and are still being used extensively in the assessment performance of optical imaging systems.

- Several workers have modified these criteria to suit various imaging situations for the propose of comparing and assessing the performance of optical systems. 
- As the Rayleigh criterion has limited applicability, we have chosen the Sparrow criterion also. The Sparrow criterion is sensitive to various parameters such as intensity ratio of object points, non-uniform transmission of the aperture, degree of coherence of illumination of the object points. The Sparrow criterion is amenable to quantitative calculations

- It has been empirically found that the effects of noise limitations on the two-point resolution correlate well with these two criteria.

\section{The Rayleigh Criterion}

The celebrated Raleigh criterion states that "the two point sources are just resolved if the maximum of one irradiance pattern coincides with the first minimum of the other". This means that two closely spaced points can be considered as just resolved if we are able to distinguish the resultant PSF in the image as being due to two objects instead of one. It may be pointed out the Rayleigh proposed his criterion to be used for line spectra in Spectroscopy. But it can be equally applied for images of point objects as well. In its original form, the Rayleigh criterion is applicable to two equally bright points under incoherent illumination. The Rayleigh criterion implies a pronounced central dip (minimum) in the resultant image intensity distribution curve of the equally bright and incoherent object points. This dip or the 'saddle point' is midway between the two PSF peaks. For circular apertures, the dip-point intensity is $73.5 \%$ of the maximum intensity. This implies a drop of $36.5 \%$ in intensity. The corresponding values for slit apertures are respectively $81.1 \%$ and $18.9 \%$, for a circular aperture the intensity distribution is the Bessel function squared or the Besinc function and for one dimensional object (slits) it is the sinc function squared.

The coherent Rayleigh limit for two dimensional systems is 5,146 dimensionless diffraction units for point objects of equal intensity. In the resultant image intensity distribution curve a smaller drop in intensity is associated with a smaller limit of resolution. Several researchers, to suit various imaging situations, modified the Rayleigh criterion. To suit the case of object points of unequal intensities TOLANSKY [6], CHATURVEDI and SODHA [7] modified the Raleigh criterion. In the redefined Rayleigh criterion, the two objects points are said to be just resolved, if the contrast between the lower intensity peak $I_{L P}$ and the dip (saddle point) point of minimum intensity $I_{D i p}$ is given by,

$$
\frac{I_{D i p}}{I_{L P}}=0.735 \quad \text { or } \frac{L_{L P}-I_{D i p}}{I_{L P}}=0.265
$$

BHATNAGAR, SIROHI and SHARMA [5] have employed the above modified Rayleigh criterion for case of unequally bright object points. The corresponding value for two lines is 0.19 .

\section{The Sparrow Criterion}

The Rayleigh criterion cannot be applied for intensity spread functions having non-zero minima or coherent systems or for unequally bright point objects. Sparrow recognized the limitations and the arbitrariness involved in the Rayleigh criterion and observed that "as originality proposed, the Rayleigh criterion was not intended as a measure of the actual limit of resolution, but rather as an index of the relative merit of different instruments". Sparrow proposed an alternate criterion of resolution, which he is called "the undulation condition". This is referred to as Sparrow criterion. According to the Sparrow criterion, two object points can just be resolved when the second derivative of the total intensity distribution in the diffraction image of the two object points, vanishes at a point midway between the respective Gaussian image points. When this condition is satisfied, the distance between the two object points gives the Sparrow limit of resolution, hereafter to be referred to as SL. According to this criterion, two object points are said to be just resolved if in the resultant intensity distribution curve, the central dip just vanishes. The separation between the object points under these conditions gives the Sparrow Limit (SL).

When the actual separation between two object points assumed to be of equal brightness, is larger than the critical limit (SL), the dip in the resultant intensity distribution curve is at the midpoint between the two Gaussian image points. As the actual separation between the object points is decreased, the dip-point decreases in its upward concavity and it just vanishes at a particular separation of the two object points. The separation between the object points under this condition of the vanishing dip gives the Sparrow limit of resolution $Z_{0}^{\prime}$.

The Rayleigh criterion implies a finite contrast in the image while Sparrow criterion leads to the limiting case of vanishingly small contrast. "In its original context, the Sparrow criterion was applied to incoherent illumination; the immediate generalization to coherent illumination is due to LUNEBERG. The case of two objects points of equal intensity is very rare in practical imaging situations. In the holographic image formation under partially coherent illumination and in the defocused image of two points in partially coherent or coherent illumination, the object points are of unequal intensities. Realizing this aspect, ASAKURA [4] 
introduced the "modified Sparrow criterion" to suit the situation of actual object points which are unequally bright. This modified Sparrow criterion is relevant in such practical imaging systems.

The "modified Sparrow criterion" states that, "the resolution is retained, when the second derivative of the image intensity distribution vanishes at a certain point $\left(Z=Z_{0}^{\prime}\right)$ between two Gaussian image points, with the condition that this point $Z_{0}^{\prime}$ should be a solution for the first derivative of the image intensity distribution becoming zero". The modified Sparrow criterion can be mathematically written as,

$$
\frac{\partial^{2} I(z)}{\partial Z^{2}} \mid a t z=z_{0}^{\prime}=0
$$

and

$$
\frac{\partial^{2} I(z)}{\partial Z^{2}} \mid a t z=z_{0}^{\prime}=0
$$

When the two object points are of equal intensities and very well separated, in the resultant intensity distribution curve, there will be a very well pronounced dip point, which is located at the centre between the Gaussian image points. When the two object points are of unequal intensities, it is noticed that the dip point in the resultant intensity distribution curve is not located midway between the two Gaussian image points. It is also observed that as the difference between the intensities of the object points increases, the dip point is found to shift towards lower peak in the intensity distribution curve.

As the two object points come closer, the dip disappears at a certain separation. This vanishing dippoint becomes a point of inflection which is no longer a minimum or maximum point. At this point, both the first and the second derivatives of the resultant intensity distribution become zero.

\section{Review Of Previous Works On TPR}

The two-point resolution is historically, one of the earliest physical parameters proposed to evaluate the performance of optical imaging systems. The various criteria of resolution that have proposed from time to time by several authors are all arbitrary, and hence, they do not determine or set absolute limit of resolution imaging system. The studies on the problem of two-point resolution of imaging systems were initiated by LORD RAYLEIGH [8]. Subsequently, this problem has attracted the attention of several researchers, several authors who have investigated this problem in various imaging situations. Hence, a vast amount of literature has been reported by several authors on this subject. The papers of CESINI et. al. [9], BARAKAT [10], ASAKURA [4] and MILLS and THOMPSON [11] provide a very good review of the studies on this subject.

The survey of the literature reveals that both the Rayleigh and Sparrow criteria were modified to suit various imaging situations, CHATURVEDI and SODHA [7], ASAKURA [4], JAISWAL and BHOGRA [12], BHATNAGAR, SIROHI and SHARMA [5], etc. CARSWELL and RICHARD [13] suggested a criterion for coherent system as an extension of Rayleigh criterion. The Rayleigh criterion was based on the tacit assumption that the two object points are incoherent and it was stated for object points of equal intensity. An alternate and more practical criterion, called the Sparrow criterion, for equally bright point object in incoherent illumination was stated by Sparrow. ASAKURA [4] introduced the modified Sparrow criterion for two point resolution of unequally bright points and investigated the Sparrow limit in partially coherent illumination.

The two-point resolution studies for one- dimensional systems have been made by ROJAK [14] for intermediate states of coherence NYYSSONEN and THOMPSON [15] have plotted and studied the actual intensity distribution in the image for the coherent and actual intensity distribution in the image for the coherent and incoherent extremes. GRIMES and THOMPSON [16] discussed the two-point resolution with partially coherent light for equally bright object points. They have studied the relation between the measurable and the real separations of the two object points and also verified it experimentally.

GUPTA, SIROHI and NAYYAR [17] used the Sparrow criterion and derived an expression to obtain the limit of resolution or an annular aperture in partially coherent light. They have also studied the variation of the critical resolution for various obscuration ratios and found a near linear relation. A few studies have also been reported on the problem of two- point resolution in microscopes. BASURAY [18] has studied the twopoint resolution of phase objects in partially coherent light in ordinary microscopes. BHATNAGAR and SIROHI [19] have studied the effect of a centrally obstructed condenser on resolution of a microscope.

MEHTA [20] employed Sparrow criterion and investigated the dependence of the critical resolution of coherent properties of the point's sources taking into account non-uniformity of illumination. He found that the non- uniform illumination has increased the just resolvable separation. MEHTA, VIRDI and NAYYAR [21] studied the two-point resolution by a circular aperture employing non- uniform and non-symmetric illumination. 
ASAKURA [4] for the first time introduced the modified Sparrow criterion to study the two-point resolution of unequally bright points under partially coherent illumination for the Airy pupil. SODHA and AGARWAL [22] discussed the dependence of the limit of resolution of telescopes on various factors like the intensity ratio, background intensity and the ratio of the minimum to the lower maximum of the resultant intensity pattern of the two objects. BHATNAGAR, SIROHI and SHARMA [5] made use of the modified Rayleigh criterion and investigated the dependence of the limit of resolution, on the intensity ratio and the background intensity in partially coherent light.

The literature is rich in the studies on the effect of apodisation on the two-point Resolution of imaging systems. BARAKAT and LEVIN [23] used apodization to increase the two-point resolution in terms of the Sparrow criterion, for both coherent and incoherent cases. ASAKURA and UENO [24] also employed apodisation to increase two-point resolution and obtained the required pupil function by solving the homogeneous Freehold integral equations. SHANKARAIAH et.al. [25] used Gaussian apodisation and studied the resolution of two unequally bright points in partially coherent illumination. NAYYAR and VERMA [26] have discussed the partially coherent two-point resolution of a Gaussian aperture making use of several resolution criteria. MAGIERA and MAGIERA [27] study the partially coherent two-point resolution by Walshtype apertures using the Sparrow criterion.

GRUBER and THOMPSON [28] have discussed the effect of apodisation in coherent imaging systems. SURENDAR et al [29] have used Lanczos' filters and studied the resolution of unequally bright points in partially coherent illumination. THOMPSON [30] has investigated the diffraction by annular apertures with semi-transparent central regions that add a uniform phase and found an improved two-point resolution. NAYYAR [31] has discussed two-point resolution employing both the Rayleigh and Sparrow criteria for the semi-transparent $\pi$-phase annular apertures and for the annulus in partially coherent illumination. NAYYAR and VERMA [26] have investigated the effect of non-uniform and non-symmetric illumination on the two-point resolution of a microscope using a semi-transparent $\pi$-phase annular aperture.

There have been a few studies NAYYAR [31] Mc KECHNIE [32] on the two-point resolution for two anti -phase coherent point objects with a theoretical prediction of an infinite degree of resolution which has been exploited in holographic spectroscopy. MILLS and THOMPSON [11] have combined apodisation and aberration and examined the Sparrow limit for spherical aberration, coma and defocus, both with and without apodisation. They employed Gaussian apodisers Mc KECHNIE [32].In this case, the value of $\gamma$ is neither 0 (incoherent Illumination) nor 1 (Perfectly Coherent Illumination), as $\gamma$ can assume any value in the range of $0<\gamma<1$ the equation ( $\mathrm{V}-\mathrm{c}$ ) will remain unchanged.

$$
I(Z)=|G(Z-B)|^{2}+\alpha|G(Z+B)|^{2}+2 \sqrt{\alpha} \cdot \gamma\left(Z_{0}\right)|G(Z-B)||G(Z+B)|
$$

Obviously, the values of $\gamma$ close to 0 will behave more like an incoherent situation, whereas, the values of $\gamma$ close to 1 will behave more like a coherent situation.

A close examination of the results obtained by computed values of intensities, shows that when the composite filter is used in the super-resolving region, the Rayleigh and Sparrow limits of resolution of this filter are found to be less than those for the apodised on the diffraction-limited systems. For this configuration of the composite filter the limit of Resolution are found to show a no-linear variation with the coherence parameter. Further, contrary to apodised and diffraction -limited systems, the analyzed the influence of defocusing on resolution employing modified Rayleigh criterion and found an increase in the resolution with defocus in incoherent illumination. The influence of partially coherent illumination and spherical aberration on microscopic resolution has been studied by SOM [33].

It has been observed by ASAKURA [4] that in two- point resolution studies there are only two measurable quantities the separation between the two peaks and the intensities of the peaks in the resultant image intensity distribution of a two-point object. For a perfect imaging system, these two quantities are normally expected to give corresponding actual quantities of the object points. However, this is not found to be true GRIMES and THOMPSON [16], GRUBER and THOMPSON [28], MILLS and THOMPSON [11]. The difference between the actual and measured separation (peak- to peak distance in the image plane) of the two object points has been called "mensuration error" MILLS and THOMPSON, [11]. They have also found that apodisation decreases this error, at the same time, degrade the resolution limit. They had also performed an experiment which confirms the theoretical results.

\section{Formulation of Two Point Resolution}

Our derivation will be based on the method by HOPKINS and BARHAM [34]. According to them, coherence between the two points in the object plane must be exactly the same when the condensers have the same numerical aperture for the two possible kinds of illumination viz., Kohler and critical illumination. In 
critical illumination, any two points in the object plane are illuminated co-physically but with different amplitude by a single element of the illuminant. On the other hand, in Kohler illumination, the coherent light from an element of the source illuminates the two points in the object plane with a different phase, but with the same amplitude. HOPKINS and BARHAM [34] had shown that precisely the same results are obtained for both critical and Kohler illumination. This implies that the coherence between the two points in the object plane in the two types of illumination must be the same as long as the ratio of the numerical aperture of the condenser to that of the objective is the same in both the cases as have been mentioned earlier. This conclusion was in agreement with that predicted by ZERNIKE [35]. Consequently, the expression for the total intensity distributions at an arbitrary point in the image plane remains the same irrespective of the type of illumination that is considered.

Figure 1 gives a schematic representation of the optical system for partially coherent resolution of two unequally bright object points. The relative positions of the source plane, object, the image plane and also the positions of the condenser and objective are indicated in the figure. Let us consider two- pinholes in the object plane which are illuminated by a condenser of circular aperture. The image forming objective also consists of an aperture which is also circular in shape.

It has to be mentioned that hereafter, we are going to necessarily introduce some variables whose symbols are the same for the variables used in the Ref [36]. Further, the same variables elsewhere may have different symbols. This has been necessitated because of the large number of variables used in the present work and also to retain the familiar symbols of these variables used in the most of the standard reference books and literatures. Fortunately, there will be no confusion at all, so we can safely ignore all the symbols for different variables used in other works.

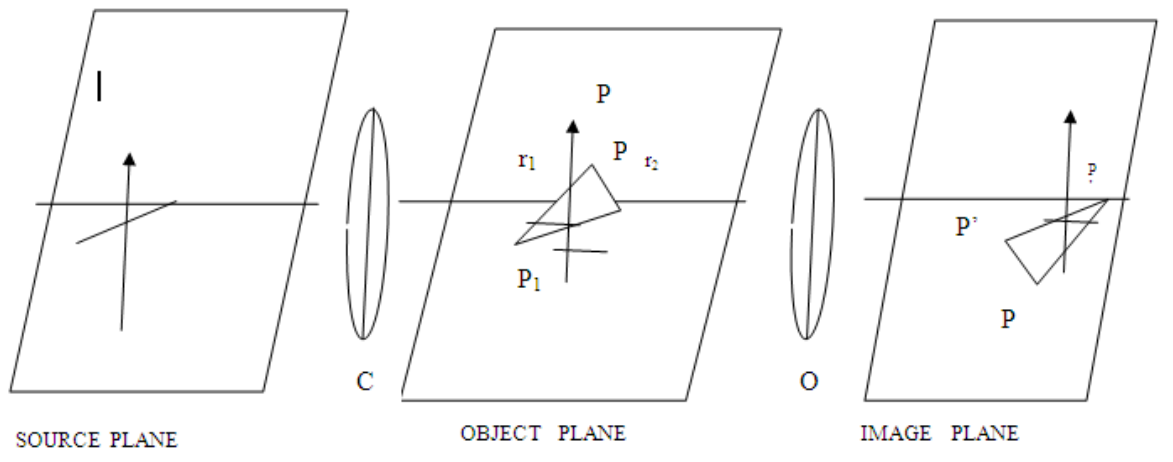

Fig.1. Optical system for the Resolution of two unequal bright points

Assuming critical illumination of the object plane, the resolvable separation between the two pin-holes can be written as

$$
d=\frac{K \lambda}{n \sin \theta}
$$

where $\theta$ is the semi-angular aperture of the objective, $K$ is a constant which is a function of $\rho$, the ratio of numerical apertures of the condenser and the objective

i.e.

$$
\rho=\frac{(N \cdot A)_{c}}{(N \cdot A)_{0}}
$$

When $\rho \rightarrow 0$ the illumination corresponds to complete coherence and when $\rho \rightarrow \infty$, it corresponds to complete incoherence of the object plane. A finite value of $\rho$ indicates that the illuminating beam is partially coherent. The magnification, $m$ of the optical system can be expressed by

$$
m=\frac{d^{1}}{d}
$$

Where $d^{1}$ is the length in the image space whose refractive index is $n^{1}$ and $d$ is the length in the object space and its refractive index is $n$. If the ray makes angles $\theta$ and $\theta^{1}$, the magnification becomes 


$$
m=\frac{n \cdot \operatorname{Sin} \theta}{n^{1} \cdot \operatorname{Sin} \theta^{1}}
$$

Further, the variable appearing in the amplitude distributions in the diffracted pattern in the image plane is the co-ordinate distance $\mathrm{Z}$ and is given by

$$
Z=\frac{2 \pi}{\lambda} d n \cdot \operatorname{Sin} \theta=\frac{2 \pi}{\lambda} d^{1} n^{1} \cdot \operatorname{Sin} \theta^{1}
$$

and the theoretical separation between the two pin holes $P_{1}$ and $P_{2}$ is given by

$$
Z_{0}=\frac{2 \pi}{\lambda} L_{0}(N \cdot A)_{0}
$$

Where $P_{1}$ and $P_{2}$ are the two apertures $\left(p_{1} p_{2}=L_{0}\right)$ whose images are $p_{1}^{1}$. Assuming an element ds of the source $\mathrm{S}$, it is imaged by the condenser $\mathrm{C}$ at $P(Z, \phi)$, its amplitude in the image of ds will be similar to that in the Airy disc with the centre at $P$. Let $P P_{1}=L_{1}$ and $P P_{2}=L_{2}$, thus the amplitude at $P_{1}$ due to dS may be written as

$$
\begin{aligned}
& A_{1}=\frac{J_{1}\left[\left(\frac{2 \pi}{\lambda}\right) \cdot L_{1}(N \cdot A)_{c}\right]}{\left[\left(\frac{2 \pi}{\lambda}\right) \cdot L_{1}(N \cdot A)_{c}\right]} \\
& =\frac{J_{1}\left(\rho r_{1}\right)}{\left(\rho r_{1}\right)} \ldots \ldots \ldots \ldots . . .(7)
\end{aligned}
$$

Where $r_{1}=\left(\frac{2 \pi}{\lambda}\right) \cdot L_{1}(N A)$ and $\sigma=\frac{(N \cdot A)_{c}}{(N . A)_{0}}$ and the amplitude at $P_{2}$ is given by

$$
\begin{aligned}
& A_{2}=\frac{J_{1}\left(\rho r_{2}\right)}{\left(\rho r_{2}\right)} \ldots \ldots \ldots \ldots \ldots(8) \\
& \text { Where } r_{2}=\left(\frac{2 \pi}{\lambda}\right) \cdot L_{2}(N A)_{0}
\end{aligned}
$$

If $\alpha$ is the ratio between the intensities of two point objects $P_{1}$ and $P_{2}(0<\alpha<1)$, then we consider $A_{2}=P_{1} \sqrt{\alpha}$.

The diffraction images of the point objects $P_{1}$ and $P_{2}$ will be formed $P_{1}^{\prime}$ and $P_{2}^{\prime}$ whose optical distances are $Z_{1}$ and $Z_{2}$ from $P^{1}$ respectively and hence the resultant amplitude at $P^{1}$ will be given by

$$
A=A_{1} \frac{J_{1}\left(Z_{1}\right)}{Z_{1}}+A_{2} \frac{J_{2}\left(Z_{2}\right)}{Z_{2}}
$$

But when the objective is apodised by a pupil, each point gives rise to diffraction image whose normalized amplitude response to unit amplitude in the object point is given by

$$
G(Z)=2 \int_{0}^{1} f(r) . . J_{0}(Z r) r d r
$$

Obviously, the intensity at $P^{1}$ due to the entire source $\mathrm{S}$ is derived by integrating $A^{2}$ over the domain $S_{1}$ and is given by

$$
\begin{gathered}
I\left(Z_{1}, Z_{2}\right)=\int_{s}\left[A_{1} G\left(Z_{1}\right)+A_{2} G\left(Z_{2}\right)\right]^{2} d s \\
=\int_{s} A_{1}^{2} G^{2}\left(Z_{1}\right) d s+\int_{s} A_{2}^{2} G^{2}\left(Z_{2}\right) d s+\int_{s} 2 A_{1} A_{2} G\left(Z_{1}\right) G\left(Z_{2}\right) d s
\end{gathered}
$$


Since the domain of $S$ is of infinite extent, the geometrical image of the source will be large compared to the distances of $P_{1}$ and $P_{2}$.

Assuming the co-ordinates of the points $P_{1}$ and $P_{2}$ as $(-Y, 0)$ and $(Y, 0)$ respectively and $2 Y=Z_{0}(Z, \phi)$ as the co-ordinates of $P$, we can write

$r_{1}^{2}=Y^{2}+Z^{2}+2 Y Z$
and $\quad r_{2}^{2}=Y^{2}+Z^{2}+2 Y Z \cdot \operatorname{Cos} \phi$

$$
\int_{s} A_{1}^{2} d s=\int_{0}^{\infty} Z \cdot d z \int_{0}^{2 \pi}\left[\frac{J_{1} \rho\left(Y^{2}+Z^{2}+2 Y Z \cdot \operatorname{Cos} \phi\right)^{\frac{1}{2}}}{J_{1} \rho\left(Y^{2}+Z^{2}+2 y z \cdot \operatorname{Cos} \phi\right)^{\frac{1}{2}}}\right] d \phi
$$

When the origin is displaced to the point $(-y, 0)$ the integration remains un-changed as the domain of the integration extended to infinity. Therefore,

$$
\begin{array}{r}
\int_{s} A_{1}^{2} d s=\int_{0}^{\infty}\left[\frac{J_{1}(\sigma \cdot Z)^{2}}{\sigma \cdot Z} \cdot d z \cdot \int_{0}^{2 \pi} d \phi=\frac{\pi}{\sigma^{2}} .\right. \\
\text { Since } \int_{0}^{\infty}\left[\frac{J_{1}(x)}{x} \cdot d x=\frac{1}{2}\right.
\end{array}
$$

on similar lines, we can write $\int_{s} A_{1}^{2} d s=\frac{\pi}{\sigma^{2}} \alpha$

$$
\begin{gathered}
\text { since } A_{2}=A_{1} \sqrt{\alpha} \\
\int_{s} A_{1} A_{2} d s=\int_{0}^{\infty} Z \cdot d z \cdot \int_{0}^{2 \pi}\left[\frac{J_{1} \sigma\left(Y^{2}+Z^{2}+2 Y Z \cdot \operatorname{Cos} \phi\right)^{\frac{1}{2}}}{\sigma\left(Y^{2}+Z^{2}+2 y z \cdot \operatorname{Cos} \phi\right)^{\frac{1}{2}}}\right]\left[\frac{\sqrt{\alpha} J_{1} \sigma\left(Y^{2}+Z^{2}-2 Y Z \cdot \operatorname{Cos} \phi\right)^{\frac{1}{2}}}{\sigma\left(Y^{2}+Z^{2}-2 Y Z \cdot \operatorname{Cos} \phi\right)^{\frac{1}{2}}}\right] d \phi
\end{gathered}
$$

which will be reduced to, when the origin is displaced to the point $(-Y, O)$ and $2 Y$ is replaced by $Z_{0}$,

$$
\left[\int_{0}^{\infty} Z . d z \cdot \int_{0}^{2 \pi}\left[\frac{J_{1}(\sigma . Z)}{\sigma . Z} \sqrt{\alpha} \frac{J_{1} \sigma\left(Z_{0}^{2}+Z^{2}-2 Z_{0} Z \cdot \operatorname{Cos} \phi\right)^{\frac{1}{2}}}{\sigma\left(Z_{0}^{2}+Z^{2}-2 Z_{0} Z \cdot \operatorname{Cos} \phi\right)^{\frac{1}{2}}}\right] d \phi\right.
$$

using Newman's addition theorem for Bessel functions the eqn. (18) can be expanded as

$$
\begin{array}{r}
J_{0}(Q)=J_{0}(a) J_{0}(b)+2 \sum_{p=1}^{\infty} \operatorname{Cos}(P \phi) J_{p}(a) J_{p}(b) \\
\text { where } Q=\left(a^{2}+b^{2}-2 a b \operatorname{Cos} \phi\right)^{2}
\end{array}
$$

Differentiating the eqn. (19) with respect to $Q$ we obtain

$$
\begin{aligned}
& \frac{\partial}{\partial Q}\left[J_{0}(Q)\right]=\frac{-J_{1}(Q)}{Q} a b . \operatorname{Sin} \phi \ldots \ldots \ldots \ldots(20) \\
& \text { Thus, } \quad \frac{-J_{1}(Q)}{Q} a b \sin \phi=-2 \sum_{p=1}^{\infty} P . \operatorname{Sin}(P \phi) J_{p}(b)
\end{aligned}
$$




$$
\frac{J_{1}(Q)}{Q} b=\left(\frac{2}{a}\right) \sum_{p=1}^{\infty} \frac{P \operatorname{Sin} P \phi}{\operatorname{Sin} \phi} J_{p}(a) J_{p}(b)
$$

Assuming $a=\sigma Z_{0}, b=\sigma Z_{0}$, and Eqn.(5.20), the Eqn. ( 5.18) can be written as

$$
\int_{s} A_{1} A_{2} d s=\frac{2 \sqrt{\alpha}}{\sigma^{2} Z_{0}} \int_{0}^{\infty} \frac{J_{1}(\sigma Z)}{Z} \cdot d Z \int_{0}^{2 \pi} \sum_{p=1}^{\infty} \frac{P \operatorname{Sin}(P \phi)}{\operatorname{Sin} \phi} J_{p}(a) J_{p}(b) d \phi .
$$

Where $\phi$ takes the value of $2 \pi$, when $P$ is odd and $\phi$ takes the value of zero, when $P$ is even. Thus, Eqn (5.21) becomes

$$
\int_{s} A_{1} A_{2} d s=\frac{4 \pi \sqrt{\alpha}}{\sigma^{2}} \sum_{p=13 \ldots}^{\infty} \frac{J_{p}\left(\sigma . Z_{0}\right)}{\sigma . Z_{0}} \int_{0}^{\infty} \frac{J_{1}(\sigma . Z) J_{p}(\sigma . Z)}{Z} d Z
$$

The integral in Eqn (5.22) is one of the Lommel's integrals. Its value is $\frac{1}{2}$ for $P=1$ and zero for other odd integral values.

Thus,

$$
\int_{s} A_{1} A_{2} d s=\frac{4 \sqrt{\alpha}}{\sigma^{2}} \frac{2 J_{1}\left(\sigma \cdot Z_{0}\right)}{\left(\sigma \cdot Z_{0}\right)}
$$

The total intensity at $p^{1}$ in the image plane will be obtained by substituting the values from the Eqns (15), (16), (23) in Eqn. (11) as

$$
I\left(Z_{1} Z_{2}\right)=\frac{\pi}{\sigma^{2}} G^{2}\left(Z_{1}\right)+\frac{\pi \alpha}{\sigma^{2}} G^{2}\left(Z_{2}\right)+\frac{2 \pi \sqrt{\alpha}}{\sigma^{2}} \frac{2 J_{1}\left(\sigma Z_{0}\right)}{\sigma Z_{0}}\left[G\left(Z_{1}\right) G\left(Z_{2}\right)\right]
$$

and is written, after ignoring $\frac{\pi}{\sigma^{2}}$ as

$$
\begin{aligned}
I\left(Z_{1} Z_{2}\right) & =G^{2}\left(Z_{1}\right)+\alpha \cdot G^{2}\left(Z_{2}\right)+\sqrt{\alpha} 2.2 \frac{J_{1}\left(\sigma \cdot Z_{0}\right)}{\sigma \cdot Z_{0}}\left[G\left(Z_{1}\right) G\left(Z_{2}\right) \ldots .\right. \\
= & G^{2}\left(Z_{1}\right)+\alpha \cdot G^{2}\left(Z_{2}\right)+2 \gamma Z_{0} \sqrt{\alpha}\left[G\left(Z_{1}\right) G\left(Z_{2}\right)\right] \ldots \ldots \ldots \ldots \ldots . . .
\end{aligned}
$$

Where $\gamma\left(Z_{0}\right)=\frac{2 J_{1}\left(\sigma Z_{0}\right)}{\sigma Z_{0}}$ is the coherence factor between the two object points. When $\sigma Z_{0} \neq 0, J_{1}\left(\sigma Z_{0}\right)=0$; then $\sigma Z_{0}$ is a root of $J_{1}\left(\sigma Z_{0}\right)$. The above condition holds good when $p_{1}$ and $p_{2}$ are incoherently illuminated. Therefore, the Eqn. (25) reduces to

$$
I\left(Z_{1} Z_{2}\right)=G^{2}\left(Z_{1}\right)+\alpha \cdot G^{2}\left(Z_{2}\right)
$$

which is particularly incoherent if $\sigma=1 ; z_{0}$ is a non-zero root of $J_{1}\left(\sigma Z_{0}\right)=0$, that is, the distance between the two point objects $p_{1}$ and $p_{2}$ equal to the radius of the every dark ring of the Airy pattern. The resolution of these point objects, incoherently illuminated if $(N . A)_{0}=(N . A)_{c}$, is given by

$$
d=\frac{0.61 \lambda}{n \sin \theta}
$$

This is possible only when $Y\left(Z_{0}\right)=0$ and the two narrow apertures are illuminated, incoherently. When the aperture of the condenser is made limiting narrow i.e. $\sigma \rightarrow 0$

$$
\begin{array}{r}
\text { and } \frac{2 J_{1}\left(Z_{0}\right)}{\left(\sigma Z_{0}\right)}=1 \text {, we get } \\
I\left(Z_{1} Z_{2}\right)=\left[G\left(Z_{1}\right)+\sqrt{\alpha} \cdot G\left(Z_{2}\right)\right]^{2} \ldots
\end{array}
$$


which implies that through the distance between the two points is independent of the aperture of condenser, both the points are illuminated with coherent light and is obtained when $\gamma\left(z_{0}\right)=1$ Measuring the coordinate $\mathrm{x}$ from the mid-point, the total resultant intensity along the line $p_{1}{ }^{\prime}$ and $p_{2}{ }^{\prime}$ is formulated as

$$
I(x)=I(x+y)+\alpha I(x-y)+2 \sqrt{\alpha} y\left(Z_{0}\right) G(x+y) G(x-y)
$$

where

$$
I(x+y)=|G(x+y)|^{2} ; I(x-y)=|G(x-y)|^{2} ; x=y \text { and } y=Z_{0} / 2,
$$

It is found that using Kohler illumination method, the expression (29) can also be derived. The total intensity in case of equally bright object points, by assuming different coherence factors between them, have also been obtained by GRIMES and THOMPSON [16] and HOPKINS and BARHAM [34]. Where $X=Z_{0} / 2$ and $Z_{0}$ is the separation between the object points $p_{1}$ and $p_{2}$ the expression (29) obtained above is in agreement with the expression obtained by ASAKURA [4] for the total intensity in the image of two unequally bright points in partially coherent illumination for the Airy case. Asakura's expression is given by

$$
I(X)=\left[\frac{2 J_{1}(X-B)}{(X-B)}\right]^{2}+\alpha\left[\frac{2 J_{1}(X+B)}{(X+B)}\right]^{2}+2 \gamma \sqrt{\alpha}\left[\frac{2 J_{1}(X-B)}{(X-B)}\right]\left[\frac{2 J_{1}(X+B)}{(X+B)}\right] \ldots
$$

Equation (29) is same as equation (31) with the following notation.

$$
\begin{gathered}
G\left(Z_{1}\right)=\frac{2 J_{1}(X-B)}{(X-B)} ; G\left(Z_{2}\right)=\frac{2 J_{1}(X+B)}{(X+B)} \\
2 B=Z_{0} ; \gamma=\gamma\left(Z_{0}\right)
\end{gathered}
$$

Equation (31) with a slight change of notation may be rewritten as

$$
I(Z)=|G(Z-B)|^{2}+\alpha|G(Z+B)|^{2}+2 \sqrt{\alpha} \gamma(Z)|G(Z-B)||G(Z+B)|
$$

Where $2 B=Z_{0}$ is the actual separation between the object points, $\alpha$ is the ratio of the intensities of the object points and $\gamma\left(Z_{0}\right)$ is the real part of the complex degree of coherence of illumination of the object point. $G(Z+B)$ and $G(Z-B)$ are the complex amplitude impulse response functions of the optical imaging system corresponding to the object points, each of which is situated at a distance of $Z_{0} / 2$ on either side of the optical axis. The amplitude impulse response functions $G(Z \pm B)$ are given by.

$$
G(Z \pm B)=2 \int_{0}^{1} f(r)=\alpha+\beta r^{2} J_{0}[(Z \pm B) r] d r
$$

The above expression gives the amplitude response function at the Gaussian focal plane if $f(r)=1$ the above expression gives the amplitude impulse response function for Airy pupil.

$$
G(Z \pm B)=2 \int_{0}^{1} J_{0}[(Z \pm B) r] r d r
$$

The amplitude response function at a defocused plane specified by $Y$ is given by,

$$
G[Y,(Z \pm B)]=2 \int_{0}^{1} f(r) \exp \left[-i y r^{2} / 2\right][(Z \pm B)] r d r
$$

Where $f(r)$ is the filter of the optical system and it specifies the non-uniformity of amplitude transmission at the exit pupil.

\section{Acknowledgement}

The authors are grateful to Prof.P.K.Mondal, the director, Mondal Institute of Optics(MIO), Hyderabad, Andhra pradesh, India for developing our interest in this topic. 


\section{References}

[1] BARAKAT, R., opt. Acta., vol.17, 1969.

[2] RONCHI, V., et.al., Atti. Fond. G. Ranchi., vol.35, 1980.

[3] LORD. RAYLEIGH, Collected papers (Cambridge Univ. Press, Cambridge), Vol.3, 1902.

[4] ASAKURA,T., Nouv, Rev.opt., vol.5, 1974.

[5] BHATNAGAR, G.S., SIROHI, R.S. and SHARMA, S.K., opt. commun., vol.3, 1971.

[6] TOLANSKY, S.C. "High Resolution Spectro Scopy (Mathuen \& Co., Landon), 1947.

[7] CHATURVEDI, K.C. and SODHA, M.S., Indian J. Phys., vol.30, 1956.

[8] LORD. RAYLEIGH, Collected papers (Cambridge Univ. Press, Cambridge), Vol.3, 1902.

[9] CESNI, G., et.al., J. optics (paris), vol.10, 1979.

[10] BARAKAT, R., J. opt. Soc. Am., vol.52, 1962.

[11] MILLS, J.P. and THOMPSON, B.J., J. Opt.Soc. Am.A., vol. 3, 1986

[12] JAISWAL, A.K. and BHOGRA, R.K., optica. Acta., vol.21, 1974.

[13] CARSWELL, A.I. and RICHARD, C., Appl. Opt., vol.4, 1965.

[14] ROJAK, F., M.S. THESIS, “Two point Resolution with partially coherent Light”, Lowel Technological Institute, Lowell, mass., 1961.

[15] NYYSSONEN, D. and THOMPSON, B.J., J. opt. Soc. Am., vol.57, 1967.

[16] GRIMES, D.N. and THOMPSON, B.J., J. opt. soc. Am. vol. 57, 1967.

[17] GUPTA, B.N., SIROHI, R.S. and NAYYAR, V.P., Phys.Letters.,vol.33A,1970.

[18] BASURAY, A., J. opt. India, vol.1, 1972.

[19] BHATNAGAR, G.S. and SIROHI. R.S., optica. Acta., vol.18, 1971.

[20] MEHTA, B.L., Appl. Opt., vol.13, 1974.

[21] MEHTA, B.L., VIRDI, S.P.S. and NAYYAR, V.P., Atti. Fond.G. Ronchi, vol.26,1971.

[22] SODHA, M.S. and AGARWAL, A. K., Optik, vol.24, 1967.

[23] BARAKAT, R. and LEVIN, E., J. opt. Soc. Am., vol. 53, 1963.

[24] ASAKURA, T. and UENO, T., J. Opt ( paris)., vol.8, 1977.

[25] SHANKARAIAH, M., et.al., Atti. Fond. G. Ronchi., vol.37, 1982.

[26] NAYYAR, V.P. and VERMA, N.K., Appl. Opt., vol.17, 1978

[27] MAGIERA, A. and MAGIERA, L.Optica.Applicata., vol.14, 1984.

[28] GRUBER, L.S. and THOMPSON, B.G., opt. Eng., vol.13, 1974.

[29] SURENDAR, K., et.al., opt. India, vol.22, 1993.

[30] THOMPSON, B.J., "Image assessment and Specification" (Ed. D. Dutton, Proc. SPIE, California),

[31] NAYYAR, V.P., Nouv. Rev. opt., vol.5, 1974.

[32] Mc KECHINIE, T.S., Optica. Acta., vol.20, 1973.

[33] SOM, S.C., opt. Acta., vol.18, 1971.

[34] HOPKINS, H.H. and BARHAM, P.M., Proc. Phys., 1950.

[35] ZERNIKE, F., Physica., vol. 5, 1938.

[36] LIPSON, A . LIPSON, J. and LIPSON, H ., "Optical Physics", $4^{\text {th }}$ Ed Cambridge University Press,

vol.46, 1974

London, 2011 